\title{
Pattern selection near the onset of convection in binary mixtures in cylindrical cells
}

\author{
Arantxa Alonso, Isabel Mercader and Oriol Batiste \\ Departament de Física Aplicada, Universitat Politècnica de Catalunya, Mòdul B4, \\ 08034 Barcelona, Spain \\ E-mail: arantxa@fa.upc.edu
}

Received 14 November 2013

Accepted for publication 11 February 2014

Published 17 July 2014

Communicated by M Asai and A Gelfgat

\begin{abstract}
We report numerical investigations of three-dimensional pattern formation of binary mixtures in a vertical cylindrical container heated from below. Negative separation ratio mixtures, for which the onset of convection occurs via a subcritical Hopf bifurcation, are considered. We focus on the dynamics in the neighbourhood of the initial oscillatory instability and analyze the spatiotemporal properties of the patterns for different values of the aspect ratio of the cell, $0.25 \lesssim \Gamma \lesssim 11(\Gamma \equiv R / d$, where $R$ is the radius of the cell and $d$ its height). Despite the oscillatory nature of the primary instability, for highly constrained geometries, $\Gamma \lesssim 2.5$, only pure thermal stationary modes are selected after long transients. As the aspect ratio of the cell increases, for intermediate aspect ratio cells such as $\Gamma=3$, multistability and coexistence of stationary and time-dependent patterns is observed. In highly extended cylinders, $\Gamma \approx 11$, the dynamics near the onset is completely different from the pure fluid case, and a startling diversity of confined patterns is observed. Many of these patterns are consistent with experimental observations. Remarkably, though, we have obtained persistent large amplitude highly localized states not reported previously.
\end{abstract}

\section{Introduction}

Thermal convection in binary fluids heated from below is an example of a nonlinear system that exhibits a very rich spatio-temporal behaviour when driven away from equilibrium. Twocomponent miscible liquid mixtures close to the onset of convection display a more complex spatio-temporal behaviour than the pure fluid case. The spectrum of possible convective states 
and their bifurcation properties is wider, and this is due to the effects of concentration fluctuations. These concentration fluctuations are generated by temperature gradients through the Soret effect, by which a mass flux depends not only on the concentration gradient but also on the temperature gradient. At the same time, concentration fluctuations influence the local buoyancy forces, which drive the convective flow in the first place. So there is a coupling between Soret generated concentration variations, the resulting changes in buoyancy and the mixing of the flow.

The Soret effect is quantified by the Soret coefficient (separation ratio $S$, in nondimensional form), and in this work we consider negative separation ratio mixtures $S<0$, such as ethanol and water or ${ }^{3} \mathrm{He}-{ }^{4} \mathrm{He}$. In this case, the heavier component migrates towards the hotter boundary, so the solutal buoyancy opposes the thermal one, and the onset of convection is delayed. Moreover, when solutal and thermal buoyancies compete, the interplay between the two mechanisms of instability typically gives rise to a subcritical Hopf bifurcation of the conduction state that can lead to a rich dynamical behaviour near the threshold (Kolodner 1993).

The experimental work and the numerical simulations on nearly two-dimensional (2D) systems is abundant and shows that a great variety of patterns can arise near the onset of convection from symmetry breaking bifurcations of the isotropic conduction state (e.g. Kolodner et al 1990-1995, Barten et al 1995a, 1995b, Lücke et al 1998, Batiste et al 1999-2006). Two-dimensional simulations modelling convection in rectangular cells, both with no-slip lateral boundaries and periodic boundary conditions, reveal that some of the patterns selected by the system near threshold can be (i) spatially uniform extended solutions, both stationary (SOC states) or travelling waves (TW), (ii) localized structures in which regions of fully developed convection coexist with regions of quiescent fluid in the form of steady pulses (convectons) or travelling pulses (LTW), or (iii) small-amplitude states in which erratic bursts of convection amplitude take place (dispersive chaotic states).

The high performance achieved by computers makes it possible nowadays to address fully three-dimensional (3D) computations of fluid systems. In cylindrical geometry, several numerical works devoted to the study of pure fluid Rayleigh-Bénard convection have been published in recent years, and a linear stability analysis for binary convection in a cylinder was performed for two sets of parameters (Mercader et al 1995). But to our knowledge, detailed nonlinear simulations of binary mixture convection are still unavailable. For onecomponent fluids, convective patterns including multi-roll patterns, targets and spirals had been obtained in moderate aspect ratio cells by Rüdiger and Feudel (2000), Leong (2002), Ma et al (2006) and Mercader et al (2010). Also, in a series of works motivated by the experimental results obtained by Hof et al (1999), Boronska and Tuckerman (2010a, 2010b) carried out simulations in a small aspect ratio cell, and a wide variety of steady and time-dependent flows was obtained in the vicinity of the onset of convection. A recent work by Hébert et al (2010) includes some experimental observations on pattern formation near the onset in an intermediate aspect ratio cell and a comparison with some numerical simulations.

For binary mixtures, the large difference in the characteristic times of the thermal and mass diffusivities makes numerical simulations of convection much more costly; these two very different time scales in the problem produce narrow boundary layers in the concentration field. Recently, we developed an efficient time-evolution spectral code to simulate 3D binary convection in cylindrical cells and we used it to model the experiments performed in large aspect ratio cells by Lerman et al (1993, 1996, 1999). A general agreement between the reported experimental observations and our numerical results was obtained. The dynamics within a narrow range of Rayleigh numbers around the onset of convection turned out to be 
very complex, with a clear tendency of the system to form localized structures combining domains of travelling waves and quasi-steady convection (Mercader et al 2008). In the present paper, we extend the results of that work and analyze the influence of the size of the domain by considering several small-to-moderate values of the aspect ratio of the cell. We focus on the spatio-temporal dynamics in the neighbourhood of the initial oscillatory instability and compare two-component with one-component pure fluid convection to elucidate to what extent the dynamics is governed by thermal effects or by concentration fluctuations. We do not pursue a systematic study of the bifurcation properties of the arising patterns as the Rayleigh number is increased.

This paper is organized as follows. The numerical equations and boundary conditions of binary-mixture convection, together with the main characteristics of the numerical scheme used to solve them are specified in section 2. In section 3, we show and discuss the results of the work; first, the linear stability analysis results as a function of the aspect ratio of the cell for several binary mixtures are presented, then the nonlinear results for small, moderate and large aspect ratio cells are discussed. Finally, some concluding remarks are presented in section 4 .

\section{Equations and numerical method}

We consider Boussinesq binary fluid convection in a cylinder of height $d$ and radius $R$. The radial aspect ratio of the cylinder is defined as $\Gamma=R / d$. The cylinder is heated from below, $\Delta T$ being the temperature difference between the lids, in the presence of a vertical gravity $\mathbf{g}=-g \hat{e}_{z}$. Scaling length with the height of the layer $d$, time with the vertical thermal diffusion time $d^{2} / \kappa, \kappa$ being the thermal diffusivity, and temperature with $\Delta T$, the nondimensional equations that describe the dynamics are

$$
\begin{aligned}
& \nabla \cdot \mathbf{u}=0, \\
& \partial_{t} \mathbf{u}+(\mathbf{u} \cdot \nabla) \mathbf{u}=-\nabla p+\sigma \nabla^{2} \mathbf{u}+R \sigma[(1+S) \Theta+S \eta] \hat{e}_{z} \\
& \partial_{t} \Theta+(\mathbf{u} \cdot \nabla) \Theta=w+\nabla^{2} \Theta \\
& \partial_{t} \eta+(\mathbf{u} \cdot \nabla) \eta=-\nabla^{2} \Theta+\tau \nabla^{2} \eta .
\end{aligned}
$$

Here, $\mathbf{u}=(u, v, w)$ is the velocity field in cylindrical coordinates $(r, \theta, z), \Theta$ denotes the departure of temperature from the conduction profile, $\Theta=\left(T-T_{c}\right) / \Delta T$, and $\eta$ is defined as $\eta=-\left(C-C_{c}\right) /\left(C_{0}\left(1-C_{0}\right) S_{T} \Delta T\right)-\Theta$, where $T$ and $C$ are the fields of temperature and concentration of the denser component, $T_{0}$ and $C_{0}$ are their mean values and $S_{T}$ is the Soret coefficient. Binary fluid convection is described by four dimensionless numbers, the Rayleigh number $R$, the Prandtl number $\sigma$, the Lewis number $\tau$ and the separation ratio $S$, defined as

$$
R=\frac{\alpha \Delta T g d^{3}}{\kappa \nu}, \quad \sigma=\frac{\nu}{\kappa}, \quad \tau=\frac{D}{\kappa}, \quad S=C_{0}\left(1-C_{0}\right) \frac{\beta}{\alpha} S_{T},
$$

where $\alpha$ and $\beta$ are the thermal and mass expansion coefficients, $\kappa$ and $D$ are the thermal and mass diffusivities and $\nu$ is the kinematic viscosity. The Rayleigh number is the control parameter of the system and measures the strength of the imposed temperature gradient. The Prandtl number relates momentum diffusion to heat diffusion, while the Lewis number relates concentration diffusion to heat diffusion. The separation ratio gives the coupling between the 
thermal and concentration density gradients. A negative value of $S$ indicates that the concentration density gradient opposes the thermal density gradient, and tends to stabilize the fluid layer against thermal convection. For small $\tau$ and sufficiently negative values of $S$, the onset of convection is expected to be a Hopf bifurcation to a state of oscillatory convection (Lücke et al 1998).

For the boundary conditions, we consider a no-slip, no-flux, fixed temperature boundary at the top and bottom plates, and a no-slip, no-flux, insulating boundary on the lateral wall

$$
\begin{aligned}
& \mathbf{u}=\Theta=\partial_{z} \eta=0 \quad \text { on } z=0,1 \\
& \mathbf{u}=\partial_{r} \Theta=\partial_{r} \eta=0 \quad \text { on } r=\Gamma .
\end{aligned}
$$

Equations and boundary conditions are equivariant under the group of symmetries $\mathbf{O}(2) \times \mathbf{Z}_{2}$, where $\mathbf{O}(2)$ is generated by proper rotations $R_{\alpha}$ and reflections $\kappa$ with respect to vertical planes containing the axis, while $\mathbf{Z}_{2}$ accounts for the reflections with respect to the midplane $\gamma$. These symmetries act on the fields $u, v, w, \Theta, \eta$ as follows:

$$
\begin{array}{ll}
R_{\alpha}:(r, \theta, z) \rightarrow(r, \theta+\alpha, z), & (u, v, w, \Theta, \eta) \rightarrow(u, v, w, \Theta, \eta) \\
\kappa:(r, \theta, z) \rightarrow(r,-\theta, z), & (u, v, w, \Theta, \eta) \rightarrow(u,-v, w, \Theta, \eta) \\
\gamma:(r, \theta, z) \rightarrow(r, \theta,-z), & (u, v, w, \Theta, \eta) \rightarrow(u, v,-w,-\Theta,-\eta)
\end{array}
$$

Notice that this system is equivariant under the same group of symmetries as the two dimensional thermal convection in periodic channels is. Thus, the classification of the bifurcations of the primary solutions extensively studied in the $2 \mathrm{D}$ channel by Prat et al (1995, 1998) can be directly applied to the cylinder.

As a measure of the heat transport by convection, we use the Nusselt number $\mathrm{Nu}$, defined as the ratio of heat flux through the top plate to that of the corresponding conductive solution:

$$
N u=1-\left.A^{-1} \int_{A} \partial_{z} \Theta\right|_{z=1} d A
$$

where $A$ is the area of the cylinder lids.

To estimate the contribution to the solution of each azimuthal Fourier mode $m$, we will also evaluate the kinetic energy contained on them defined by

$$
E_{k}^{m}=\int_{z=0}^{z=1} \int_{r=0}^{r=\Gamma} \mathbf{u}_{m} \cdot \mathbf{u}_{m} r d r d z .
$$

The system of equations and boundary conditions has been solved numerically using the algorithm described in Mercader et al (2010), which can be summarized as follows. To integrate the equations in time, we use the second-order time-splitting method proposed in Hugues and Randriamampianina (1998) combined with a pseudo-spectral method for the spatial discretization, Galerkin-Fourier in the azimuthal coordinate $\theta$ and Chebyshev collocation in $r$ and $z$. The radial dependence of the functions is approximated by a Chebyshev expansion between $-R$ and $R$, but forcing the proper azimuthal parity of the variables at the origin (Fornberg 1998, Trefethen 2000). The scalar field $\Theta$, the axial velocity $w$ and $\eta$ have an even parity $(\Theta(-r, \theta)=\Theta(r, \theta+\pi))$, whereas $u$ and $v$ are odd functions. To avoid including the origin in the mesh grid, we use an odd number of Gauss-Lobatto points in $r$, and we enforce the equations only in the interval $(0, R]$. We use the standard combination $u_{+}=u+i v$ and $u_{-}=u-i v$ in order to obtain, as a result of the splitting, Helmholtz equations for all the variables $\Theta, \eta, w, u_{+}$and $u_{-}$. For each Fourier mode, these equations are 
solved using a diagonalization technique in the two coordinates $r$ and $z$. The imposed parity of the functions guarantees the regularity conditions at the origin needed to solve the Helmholtz equations (Mercader et al 1991).

In this paper, the linear stability analysis of the conduction state is also performed. Estimations of the eigenvalues and eigenvectors of the linearized problem have been obtained by using Arnoldi's method. The method is applied to calculate the dominant eigenvalues of the exponential of the Jacobian, which can be trivially related to the leading eigenvalues of the Jacobian, i.e. with those of largest real part. To this end, the algorithm for the time stepping of the linearized equations has been used, since, in fact, it approximates the action of the exponential transformation of the Jacobian on the solution at the previous time step. The eigensolving itself has been implemented using the ARPACK package.

In our simulations, for small and moderate aspect ratio cells, we have used 30 collocation points in the vertical direction $z, 160$ Fourier modes in the azimuthal direction, and 80 points in the radial direction. For slender cells $(\Gamma \approx 0.25)$, we have reduced the radial and azimuthal resolutions to 54 and 60 points, respectively, and have increased the vertical resolution to 60 points. When considering large aspect ratio cells $(\Gamma \approx 10)$ the resolution needs to be increased for some of the patterns up to 540 Fourier modes and 300 points in the radial direction to accurately resolve the arising structures. A time step of $5 \times 10^{-4}$ has proved to be sufficient to achieve convergence.

\section{Results}

In this section we analyze the influence of the aspect ratio of a cylindrical cell on pattern formation near the onset of convection for a binary mixture with a negative value of the separation ratio. We open the section with the results of the linear stability analysis of the conduction state as a function of the aspect ratio for three different binary mixtures with parameters (type i) $S=-0.1, \sigma=10, \tau=0.001$, (type ii) $S=-0.1, \sigma=0.6, \tau=0.003$ and (type iii) $S=-0.5, \sigma=0.6, \tau=0.003$ and compare them to the pure fluid case with $\sigma=1$ (section 3.1). Type (i) parameters are representative of water-ethanol mixture, while type (iii) correspond to $\mathrm{He}^{3}-\mathrm{He}^{4}$ mixtures. The study goes on with the discussion of the nonlinear results corresponding to several small aspect ratio cells in the range $0.5 \leqslant \Gamma \leqslant 2.5$ (section 3.2) and of a medium-size cell of aspect ratio $\Gamma=3$ (section 3.3). For these simulations we take as a reference for the parameters of the binary mixture the values $S=-0.1, \sigma=10, \tau=0.001$. Finally, we analyze the spatio-temporal dynamics arising near the onset of convection in $\Gamma=11$ and $\Gamma=10.5$ cylindrical cells (section 3.2 ), extending the results presented in Mercader et al (2008). The parameters we consider in this case are those used in one of the stability analysis included in Mercader et al (1991) $(S=-0.09, \sigma=24, \tau=0.008)$, which are very similar to the experimental values used in Lerman et al (1993, 1996, 1999). Fixing both the aspect ratio of the cell and the parameters of the mixture, the Rayleigh number will be the control parameter.

\subsection{Linear stability analysis and linear transients}

We will describe, first, the neutral stability curves of the conduction state for several binary mixtures, and compare them with the critical parameters obtained in the pure fluid case. Figure 1 displays the dependence of the critical Rayleigh number and frequency with the aspect ratio of the container for three binary mixtures of parameters (type i) $S=-0.1$, $\sigma=10, \tau=0.001$ (thin red solid line), (type ii) $S=-0.1, \sigma=0.6, \tau=0.003$ (green circles) 

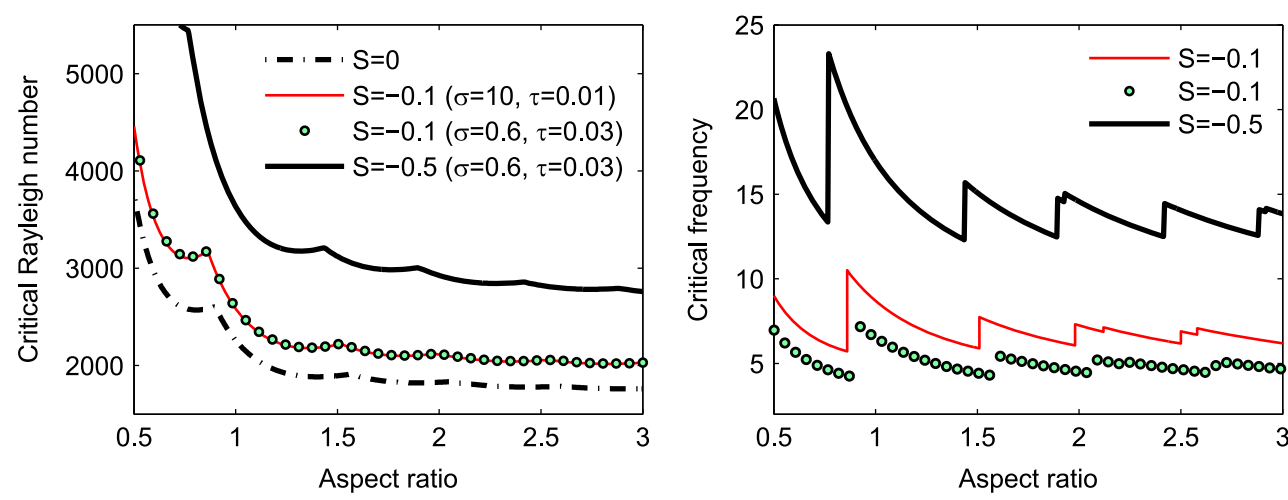

Figure 1. Critical Rayleigh number and frequency as a function of the aspect ratio for a pure fluid ( $S=0$, dashed-dotted line) and three different binary mixtures of parameters (i) $S=-0.1, \sigma=10, \tau=0.001$ (thin red solid line), (ii) $S=-0.1, \sigma=0.6, \tau=0.003$ (green circles) and $S=-0.5, \sigma=0.6, \tau=0.003$ (black thick solid line). The primary bifurcation of the conduction state is stationary for pure fluids and oscillatory for binary mixtures. All the results presented in section 3.2 and section 3.3 are obtained for a mixture with parameter values $S=-0.1, \sigma=10, \tau=0.001$.
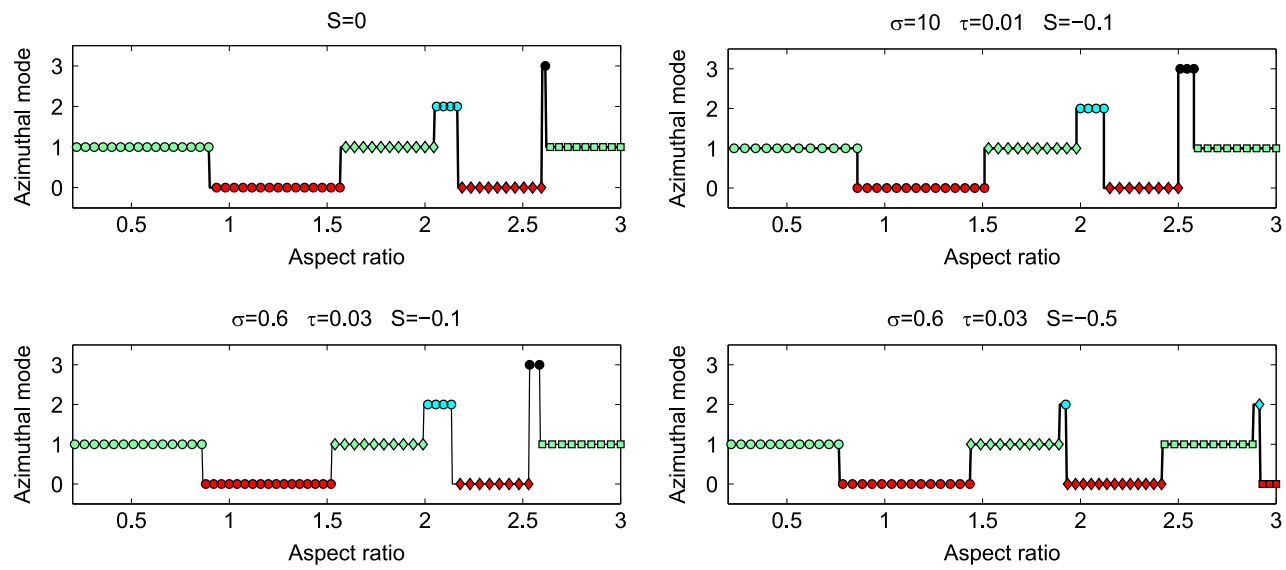

Figure 2. Selected azimuthal mode in the primary bifurcation as a function of the aspect ratio for a pure fluid and for the three binary mixtures considered in figure 1 . The markers (circle, diamond, square) indicate different radial or vertical structure of the eigenvector for the same value of the azimuthal mode.

and (type iii) $S=-0.5, \sigma=0.6, \tau=0.003$ (black thick solid line) and for a pure fluid ( $S=0$, dashed-dotted line). The results corresponding to values of the aspect ratio larger than 3 are not displayed because for such cells there is strong competition between the different azimuthal modes, and many neutral stability curves are involved in a narrow range of Rayleigh numbers.

According to our results, the separation ratio of the mixture is the most relevant parameter in the value of the critical Rayleigh number. As expected, the onset of convection is substantially delayed as the separation ratio becomes more negative, and for mixtures with the same value of $S$ and different values of the Prandtl and Lewis numbers, the value of the 
critical Rayleigh number is similar. This can be appreciated in figure 1, where the neutral stability curves for type i (red solid line) and type ii (green circles) mixtures are nearly coincident. The representation of the critical frequency included in figure 1 indicates that, while the onset of convection is stationary in the pure fluid case, the primary bifurcation for mixtures with a negative value of the separation ratio is oscillatory, even in the axisymmetric case, $m=0$. We observe that the critical frequency increases as the separation ratio becomes more negative. The value of the selected azimuthal wavenumber at the onset is represented in figure 2 as a function of the aspect ratio for the three mixtures we are considering and for the pure fluid case. The critical wavenumber seems to be mainly determined by the size of the container, especially for $\Gamma \lesssim 2.5$; however, as the aspect ratio increases, the marginal stability curves corresponding to each azimuthal wavenumber become closer to each other, and the remaining parameters of the mixture have a determining effect on wavenumber selection.

\subsection{Small aspect ratio cells}

Bearing in mind the results of the linear stability analysis, which show that the onset of convection is stationary for pure fluids and oscillatory for binary mixtures, but that the critical wavenumber is essentially the same in the two cases when the aspect ratio of the cell is small, we aim to determine the character of the bifurcation and the type of patterns selected in the neighbourhood of the onset.

To carry on the nonlinear simulations, we typically initialize the time evolution code with a slightly perturbed conductive solution consisting of a small Gaussian noise in a fixed mode of the temperature field and zero in the remaining modes and fields, and allow the system to evolve. We have performed a sequence of simulations, varying the initial state and the Rayleigh number, in order to find the asymptotic state for each cell. Since we know the exact value of the critical Rayleigh number, we have considered Rayleigh numbers slightly above onset. Figure 3 summarizes the main results obtained for small aspect ratio cells. For all the initial conditions we have tried, time evolutions always ended up in stationary solutions; we have not been able to obtain any stable time-dependent pattern in the neighbourhood of the onset of convection. Pure thermal steady modes similar to those observed in pure fluids are selected, and the concentration field does not seem to influence the dynamics. We have not observed multiplicity of solutions, the system always tends to the stationary pattern with the same azimuthal wavenumber as the critical one. As can be appreciated in figure 3, which includes a 3D representation of the temperature field, contour plots of temperature and concentration in the horizontal plane $z=0.1 d$ and a bar chart showing the contribution to the kinetic energy of each azimuthal mode, the dominant wavenumbers of the nonlinear patterns are $m=1, m=0$ (axisymmetric pattern) and $m=2$, for $\Gamma=0.25, \Gamma=1$ and $\Gamma=2$, respectively. The velocity and temperature fields are smooth, with spatial variations that are essentially harmonic, whereas there are much sharper boundary layers in the concentration field. The simulations performed with the time-evolution code show that these branches of stationary solutions are subcritical, as happens in rectangular and annular cells (Barten et al 1991). For instance, for the $\Gamma=0.25$ cell, for which the primary Hopf bifurcation takes place at $R_{c}=28191$, we have obtained that the $m=1$ steady solution is stable at least in the range of Rayleigh numbers $R \sim(25000,55500)$. In the case of the $\Gamma=1$ cell, for which the primary Hopf bifurcation takes place at $R_{c}=2584.2$, the $m=0$ steady solution is found to be stable at least within $R \sim(2408,4500)$. The fact that for $R \gtrsim R_{c}$ the system always saturates to steady patterns analogous to those obtained in pure fluid convection, rather than to timedependent patterns, indicates that the branch of oscillatory solutions is also subcritical. 


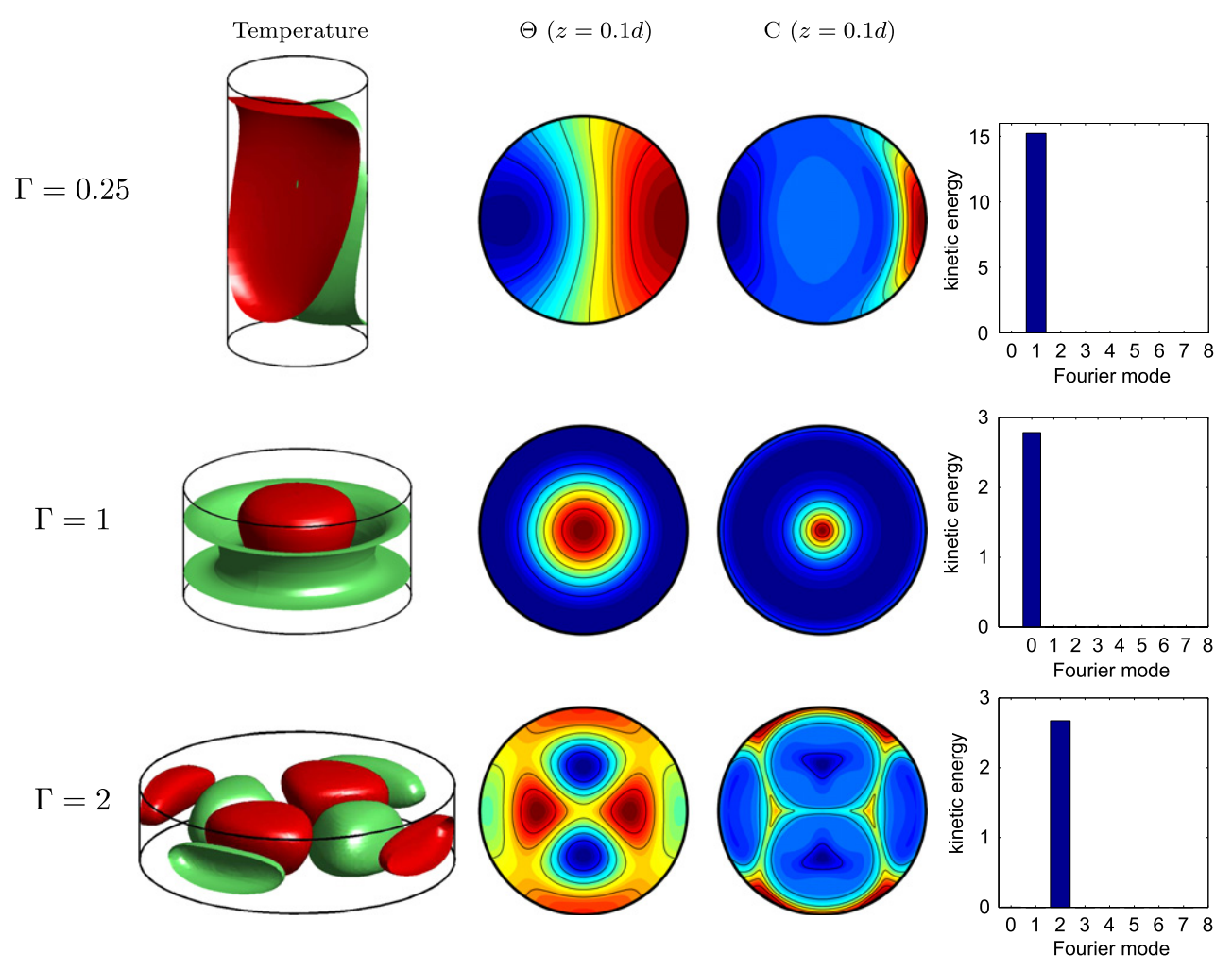

Figure 3. Nonlinear stationary patterns selected by the system in the vicinity of the onset of convection for three cells of aspect ratio $\Gamma=0.25\left(R=28200, R_{c}=28191\right)$, $\Gamma=1\left(R=2588, R_{c}=2584.2\right)$ and $\Gamma=2\left(R=2125, R_{c}=2112.6\right)$. The 3D representations correspond to temperature isosurfaces, the $2 \mathrm{D}$ plots are contour plots of temperature and concentration in a horizontal plane of height $z=0.1 d$ and the kinetic energy bar charts show the Fourier spectra in the azimuthal direction. In the three cases, the patterns are stationary, despite the oscillatory nature of the primary bifurcation, and the contribution comes exclusively from a single azimuthal mode $(m=1, m=0$ and $m=2$ ), whose value coincides with the critical azimuthal wavenumber. Parameters of the mixture: $S=-0.1, \sigma=10, \tau=0.001$.

The symmetries of the solutions shown in figure 3 are the following. For $\Gamma=0.25$, the nonlinear pattern is an $m=1$ solution invariant under the following transformations: (i) reflections with respect to an appropriate vertical plane $\theta=\theta_{0}$, denoted by $\kappa_{\theta_{0}}$, where $\kappa_{\theta_{0}}=R_{\theta_{0}} \kappa R_{-\theta_{0}}$, (ii) midplane reflection combined with a rotation of $\pi, \gamma R_{\pi}$, and (iii) the combination of both transformations, $\kappa_{\theta_{0}} \gamma R_{\pi}$. The axisymmetric pattern is invariant under (i) reflections in arbitrary vertical planes $\kappa_{\theta_{0}}$ (that is, there is no velocity in the azimuthal direction, $v=0$ ) and (ii) rotations of an arbitrary angle $\alpha, R_{\alpha}$. Finally, the solution generated by an $m=2$ mode obtained for $\Gamma=2$ is invariant under (i) rotations of $\pi(\alpha=2 \pi / m)$, (ii) reflections with respect to an appropriate vertical plane $\kappa_{\theta_{0}}$ (in this case there are two orthogonal planes that accomplish this), (iii) the combination of midplane reflection $\gamma$ with a rotation of $\pi / 2, \gamma R_{\pi / 2}$, and (iv) the combination $\kappa_{\theta_{0}} \gamma R_{\pi / 2}$. 


\subsection{Intermediate aspect ratio cells}

For a moderate aspect ratio cell, a cell of $\Gamma=3$ for which the critical Rayleigh number is $R_{c}=2028$ and the critical wavenumber is $m=1$, we obtain a different behaviour. The concentration field begins to play a role in the dynamics, and we have identified three different stable solutions very near the onset of convection. Two of these solutions are stationary and the third one is time-dependent. The three solutions are observed to be stable simultaneously in a certain range of Rayleigh numbers.

To initiate the explorations we fix the value of the Rayleigh number to $R=2031$ and introduce thermal noise in different azimuthal modes. We observe that the linear transients close to the bifurcation point can be extremely long in this case, and during these transients we can visualize different long-lasting transient states associated to the Hopf bifurcation. For example, the upper part of figure 4 shows a pattern whose spatiotemporal structure is reminiscent of an $m=1$ rotating travelling wave, which was obtained when starting the simulations with thermal noise in the $m=0$ mode. Such an $m=1$ spiral mode corresponds to one of the eigenfunctions described in Mercader et al (1995), where the linear stability analysis for cylinders of $\Gamma=2.76$ and $\Gamma=11$ was performed. The lower part of figure 4 shows a transient state similar to an $m=2$ wave travelling radially outwards, which resulted from an initial condition consisting of thermal noise in the $m=2$ mode. However, despite remaining a very long time in these patterns, the system does not saturate to these types of solutions.

In the following, we describe the three stable patterns we have been able to observe near the onset; all of them are stable for $R=2031$. To begin, we have identified a stationary fourroll solution represented in figure 5 . This pattern consists of nearly straight bars, where the main contribution of the azimuthal modes comes from the $m=4$ and $m=2$ modes, as shown in the bar chart included in figure 5. This solution is invariant under rotations of $\pi, R_{\pi}$, and under reflections with respect to two orthogonal vertical planes $\kappa_{\theta_{0}}$, but does not have any symmetry involving the midplane. This steady-bar solution is found to be stable in a range of Rayleigh numbers of $R \in(1970,2500)$. Patterns in the form of straight rolls are one of the patterns observed near the onset in pure fluid convection in cylinders, as discussed in the numerical works of Rüdiger and Feudel (2000) and Mercader et al (2010) for a $\Gamma=4$ cell and Boronska and Tuckerman (2010a, 2010b) for a $\Gamma=2$ cell.

Partially coexisting with the four-roll pattern, a different asymptotic stationary state which we call the sad-face state (shown in figure 6) has also been identified. It is a mixed mode solution where the main contribution comes from the $m=1$ and $m=4$ azimuthal modes. It is only invariant under reflections under an appropriate vertical plane $\kappa_{\theta_{0}}$. Using the sad-face state obtained for $R=2031$ as initial condition and increasing and decreasing the Rayleigh number we obtain that this state is stable for $R \in(2000,2100)$, a relatively narrow range of Rayleigh numbers. Like the four-roll pattern, this state is stable also for subcritical values of the Rayleigh number.

The time-dependent solution selected near the threshold of convection is a quasi-periodic sad-face solution. The upper part of figure 7 shows a detail of the time series for three variables of this solution, the Nusselt number and the real parts of the $m=1$ and $m=3$ vertical components of the velocity field. Although not shown, the time series for this solution is much longer, it arrives until $t \approx 5000$ thermal time units, which requires very long computational runs. The quasi-periodic nature of the solution can be inferred from the phase map and the Poincare section included in the lower part of figure 7. This figure also includes the kinetic energy bar chart, which is analogous to the stationary sad-face solution, that is, the 

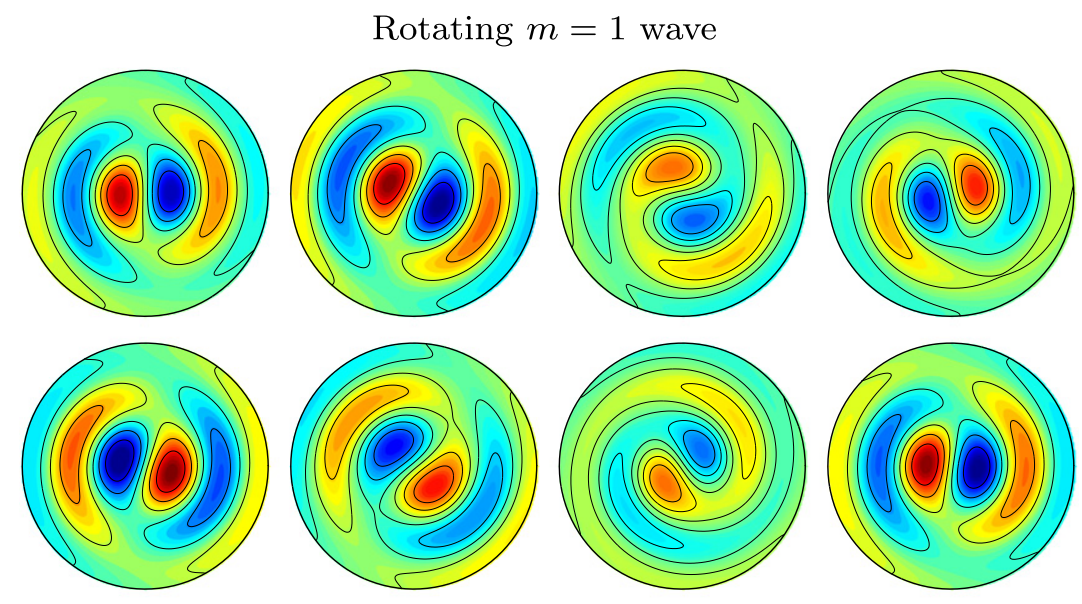

Radially outwards travelling $m=2$ wave


Figure 4. Contour plots of temperature at mid-height during two different linear transients for a $\Gamma=3$ cell at $R=2031\left(R_{c}=2028\right)$ showing patterns with a structure reminiscent of (top) an $m=1$ rotating wave and (bottom) an $m=2$ wave travelling radially outwards. Simulations are initiated with thermal noise in different azimuthal modes. After extremely long transients, the system saturates to patterns with a different spatio-temporal structure. Parameters of the mixture: $S=-0.1, \sigma=10, \tau=0.001$.

energy is mainly concentrated in the $m=1$ and $m=4$ azimuthal modes. The solution shown in figure 7 has been obtained for a subcritical value of the Rayleigh number $R=1600$ $\left(R_{c}=2031\right)$ and we have found this quasi-periodic pattern to be stable in a range of Rayleigh numbers $R \in(1935,2100)$.

To visualize the spatial variations taking place in the pattern during a pseudo-period, we have included in figure 8 the contour plots of temperature in several time-instants. The rolls break apart and join together several times within an oscillation, but superimposed to these periodic variations in the spatial structure of the pattern there is a very slow clockwise rotation. After one pseudo-period the pattern is slightly rotated, forming a modulated rotating travelling wave. Such a stable time-dependent pattern, as far as we know, has never been observed in pure fluid convection near onset. 




Figure 5. Stationary four-roll solution. Nonlinear stationary pattern selected by the system in the vicinity of the onset of convection $\left(R=2000, R_{c}=2028\right)$ for a cell of aspect ratio $\Gamma=3$ at $R=2031$. The 3D representations correspond to temperature isosurfaces, the 2D plots are contour plots of temperature and concentration in a horizontal plane of height $z=0.1 d$ and the kinetic energy bar charts show the Fourier spectra in the azimuthal direction. Parameters of the mixture: $S=-0.1$, $\sigma=10, \tau=0.001$.
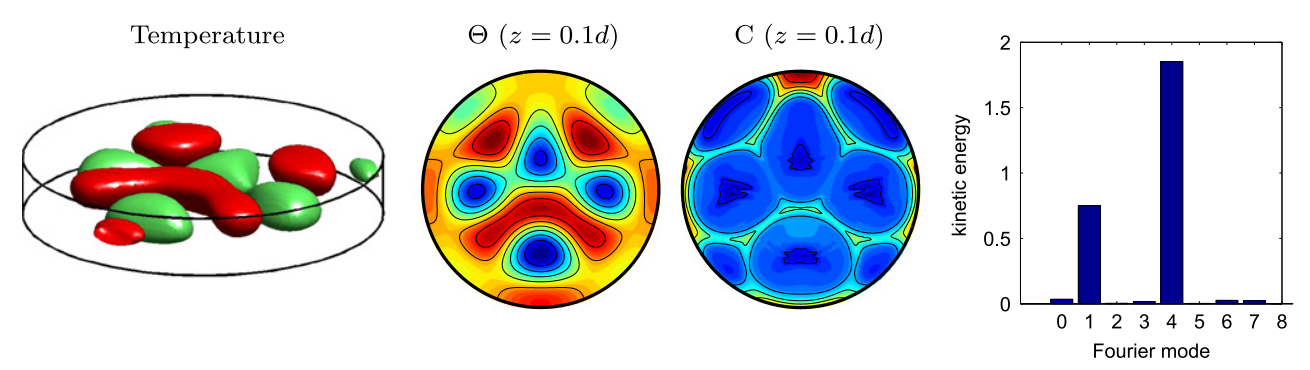

Figure 6. Stationary sad-face state. Nonlinear stationary pattern selected by the system in the vicinity of the onset of convection $\left(R=2031, R_{c}=2028\right)$ for cells of aspect ratio $\Gamma=3$. The $3 \mathrm{D}$ representations correspond to temperature isosurfaces, the $2 \mathrm{D}$ plots are contour plots of temperature and concentration in a horizontal plane of height $z=0.1 d$ and the kinetic energy bar charts show the Fourier spectra in the azimuthal direction. The pattern is a mixed-mode solution with main contribution coming from the $m=1$ and $m=4$ azimuthal modes. Parameters of the mixture: $S=-0.1, \sigma=10, \tau=0.001$.

\subsection{Large aspect ratio cells}

We present now the numerical results for a $S=-0.09, \sigma=24$ water-ethanol mixture in cells of aspect ratio $\Gamma=11$ and $\Gamma=10.5$. The choice of parameters in this paper is motivated by the experiments performed by Lerman et al $(1993,1996,1999)$ on $S \approx-0.08$ mixtures in cylindrical cells of aspect ratio $\Gamma=10.91$ and $\Gamma=11.53$. Although this system was already considered in a previous paper (Mercader et al 2008), we summarize here the main features of the dynamics described in detail in that work and incorporate some new results that complete the study.

In large aspect ratio cells the linear evolution is strongly influenced by the aspect ratio of the cell: while odd azimuthal Fourier modes dominate the dynamics in the $\Gamma=11$ cell $\left(R a_{c}=1916.2, m=1\right)$, even modes control the early stages of evolution in the $\Gamma=10.5$ case $\left(R a_{c}=1917.4, m=0\right)$. However, modes with wavenumber higher than the critical one tend to grow at much faster rates and dominate the nonlinear regime, a fact also observed in the experiments of Lerman et al (1999). 

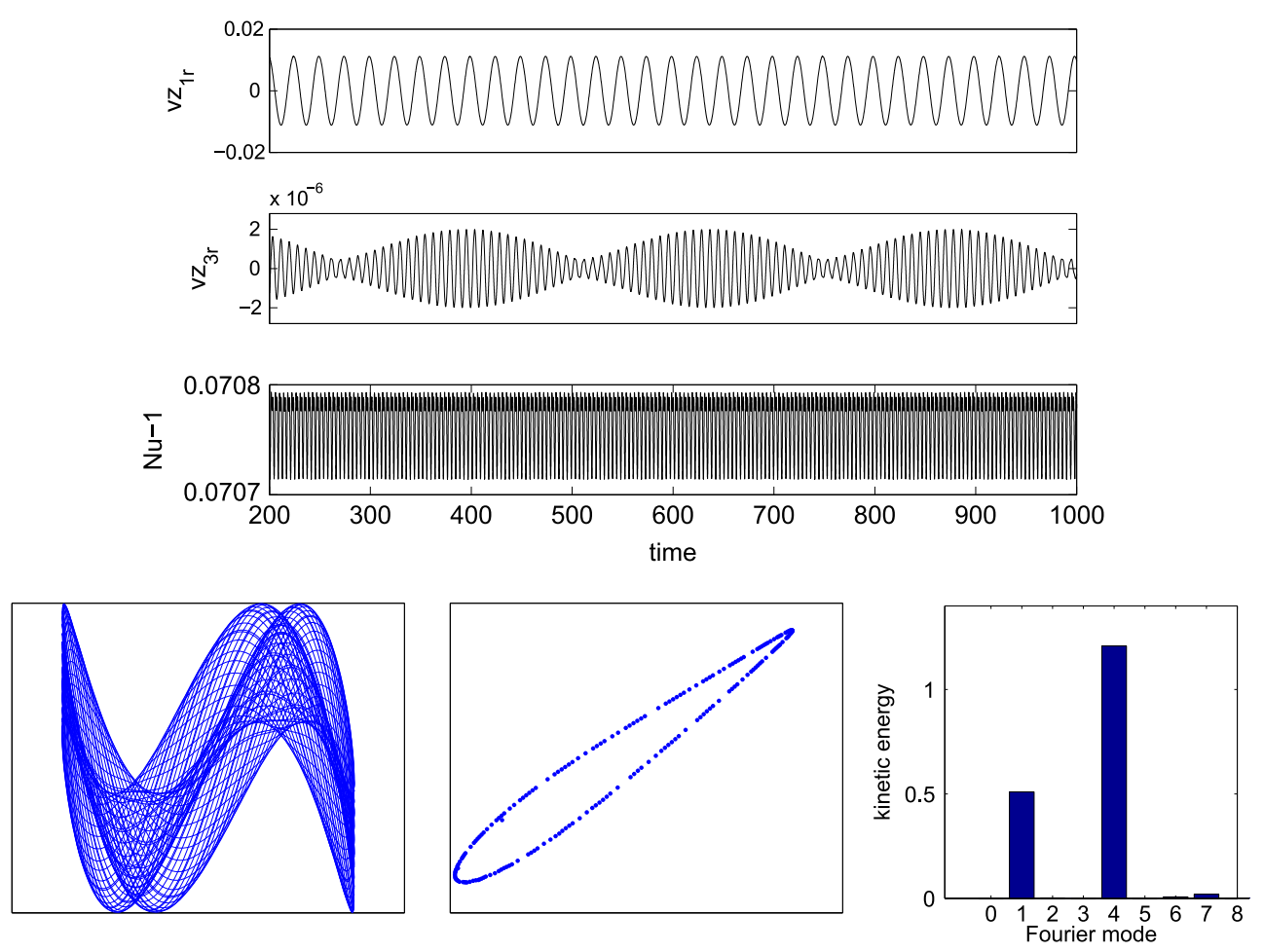

Figure 7. Quasi-periodic sad-face solution. Time series of the $m=1$ and $m=3$ vertical component of velocity and of the Nusselt number, phase map, Poincaré section and kinetic energy spectrum for the time-dependent solution obtained at $R=1960$ $\left(R_{c}=2028\right)$. Parameters of the mixture: $S=-0.1, \sigma=10, \tau=0.001$.

As for the nonlinear evolution, the actual value of the Rayleigh number turns out to be crucial in determining the dynamics, and different behaviours are observed for (i) subcritical ( $R=1914,1916$ for $\Gamma=11)$ or slightly supercritical values of the control parameter ( $R=1918$ for $\Gamma=11$ and $R=1919$ for $\Gamma=10.5$ ) and for (ii) supercritical values ( $R=1924,1934$ for $\Gamma=11$ and $R=1925$ for $\Gamma=10.5$ ). The procedure to obtain the evolution of the patterns for the lower values of the Rayleigh number is the same as in experiments, that is, linear transients are initially allowed to evolve at a larger Rayleigh number and, once the critical mode reaches saturation, the control parameter is reduced.

Although the primary bifurcation is known to be subcritical, for subcritical values of the Rayleigh number convection always dies away after some bursting episodes $(R=1914,1916, \Gamma=11)$. For slightly supercritical Rayleigh numbers $(R=1918, \Gamma=11$; $R=1919, \Gamma=10.5$ ), the system can exhibit small amplitude bursting behaviour (growths and collapses of convection amplitude take place) for a long time (see figure 9), in a way that bears a strong resemblance to the dispersive chaotic states observed in large aspect ratio annular containers for small negative values of the separation ratio (Leong 2002, Kolodner et al 1995, Alonso et al 2007). Small amplitude states consisting of stripes of convection aligned along one or more cell diameters or radii are observed during the early transients for subcritical and slightly supercritical values of the control parameter. Also in this regime, when the system exhibits bursting behaviour, patterns are confined radially in pulses during the collapse stages and is reestablished during the bursting periods (see figure 10). 


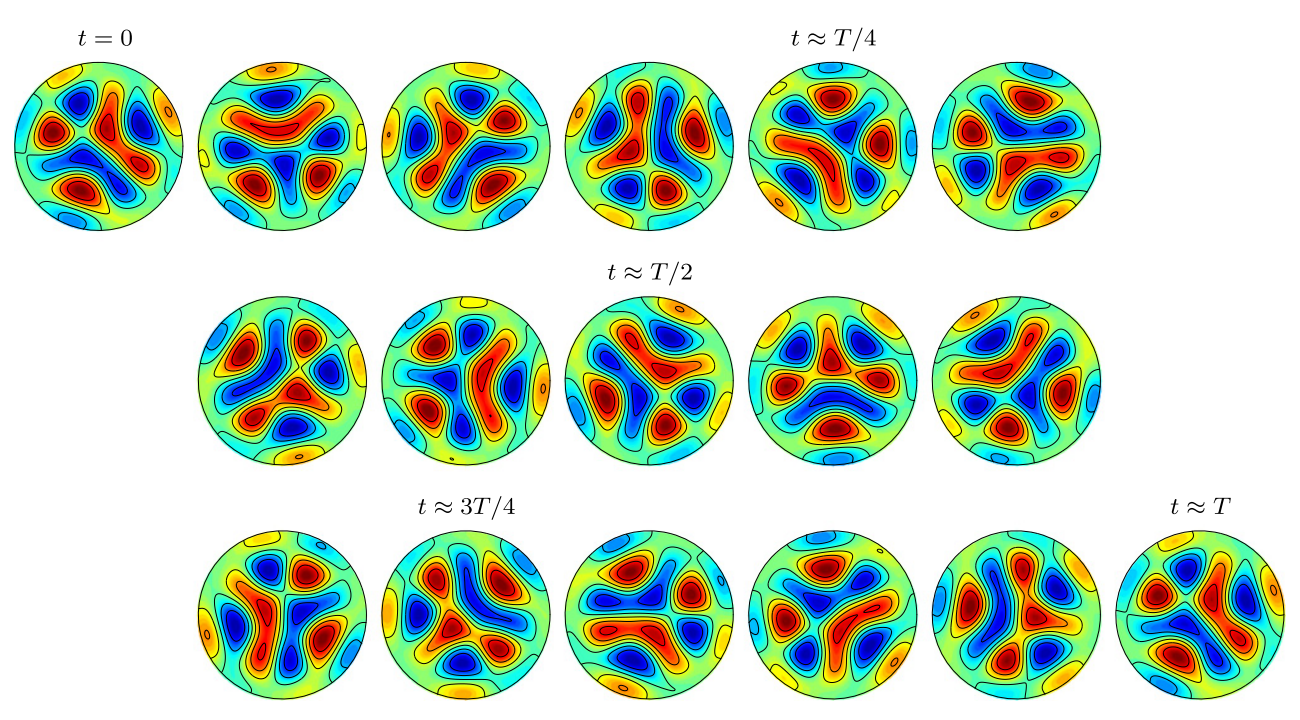

Figure 8. Quasi-periodic sad-face state. Contour plots of temperature showing at different time-instants the spatial structure of the quasi-periodic pattern selected by the system in the vicinity of the onset of convection for a cell of aspect ratio $\Gamma=3$. The pattern is a modulated rotating travelling wave. Parameters of the mixture: $S=-0.1$, $\sigma=10, \tau=0.001$.

For supercritical values, the system evolves and visits several localized states, which can either end up in persistent localized states or in extended states depending on the actual value of the Rayleigh number. In both cases, the evolution when increasing the Rayleigh number from a pattern obtained for a slightly supercritical Rayleigh number, is as follows. The Nusselt number increases progressively while growing blobs of disordered convection form around the cell center. These blobs consist of azimuthally travelling waves in a ring-shaped region in its outer part, surrounding an area of nearly stationary convection. In the case of $R=1924, \Gamma=11$ and $R=1925, \Gamma=10.5\left(\epsilon=\frac{R-R_{c}}{R_{c}} \approx 5.0 \times 10^{-3}\right)$, when the blob contacts the cylinder walls, the rolls slow down and the quiescent regions start to increase their area, causing a decrease in the Nusselt number (see figure 9), and giving rise to a wall attached confined structure (figure 11, left). Remarkably, according to our simulations, the system remains in this erratic state of localized convection with competing domains of travelling waves and quasi-steady convection. In agreement with the phenomenology described in Lücke et al (1998), in the domains of quasisteady convection the concentration field is practically homogeneous. However, such a permanent localized state does not seem to have been observed in the experiments. For a slightly larger value of the Rayleigh number $R=1934, \Gamma=11\left(\epsilon \approx 1.0 \times 10^{-2}\right)$, though, the blobs evolve filling slowly the whole cell with domains of large amplitude nearly stationary rolls (figure 11, right).

As far as the cell-filling state is concerned, after extending our time evolution calculations for the solution at $R=1934, \Gamma=11$ until $t=1800$ thermal units, which would correspond to approximately $63 h$ in a real experiment, and having increased notably the grid size, we can conclude that a strictly stationary state is never reached in the system, in contrast to the results reported in the experiments of Lerman et al (1999). However, the motion of the pattern we observe is rather slow. To visualize these variations in the pattern, we show in the left part of figure 12 the contour plots of the temperature field in the mid-plane in several time instants. Unlike the steady state of straight rolls limited by square cells displayed in the work of 

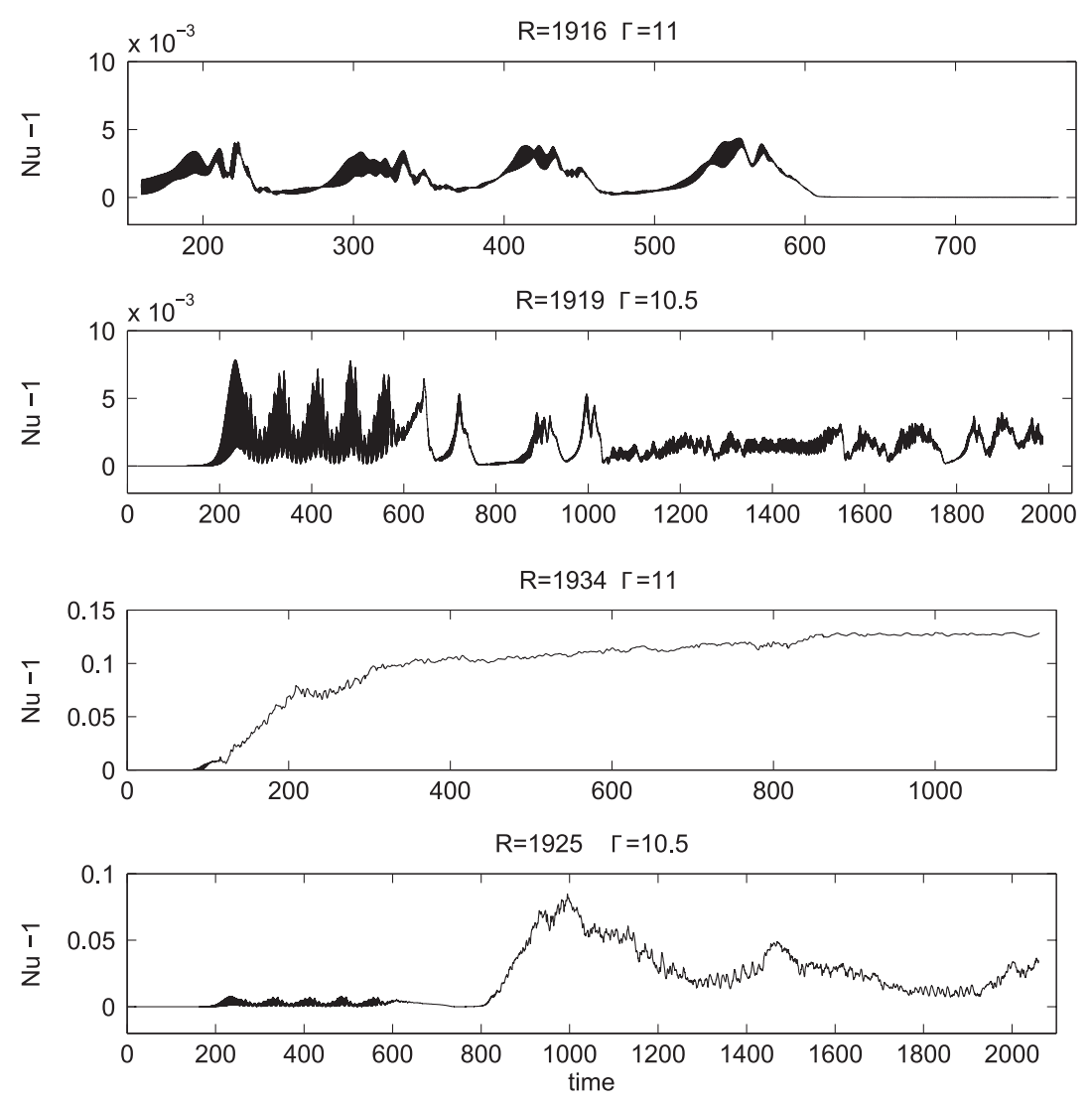

Figure 9. Time series (Nusselt number versus time) showing the amplitude of convection for different solutions during the nonlinear evolution for (i) subcritical $(R=1916, \quad \Gamma=11)$ and slightly supercritical $(R=1919, \quad \Gamma=10.5)$ values of $R$ and (ii) supercritical $(R=1934, \Gamma=11$ and $R=1925, \Gamma=10.5)$ values of $R$.

Mercader et al (2008) (figure 7) for a pure fluid $(S=0)$, in the binary mixture we observe a slow-moving structure formed by bent rolls or oblique regions of straight rolls separated by a straight line defect. In agreement with the slow movement of the bulk, the concentration field is practically homogeneous except in the narrow layers between adjacent rolls. Near the walls though, the difference of concentration values in contiguous rolls increases and quicker oscillations of the concentration field are observed (see concentration field in figure 11, right). To visualize the different behaviours, we have plotted in the right part of figure 12 the values of temperature deviation at two points of the cell as a function of time. While temperature in a point located close to the lateral boundaries, at $r=0.85 R$, exhibits clear oscillations (solid line), the value of temperature within the bulk, at $r=0.47 R$, presents non-oscillatory variations associated to the slow winding movement of the rolls (dashed line).

Another aspect of the dynamics that is worth emphasizing and that we have recently observed, is the existence of a hysteretic region in which the small amplitude states that exhibit bursting behaviour coexist with higher amplitude localized states. If we take as initial condition an almost filled state and decrease the Rayleigh number until a value in which the persistent small amplitude states exist, we obtain a higher amplitude erratic confined state similar to the state in the left part of figure 11. We show what these two coexisting solutions look like at a particular time 


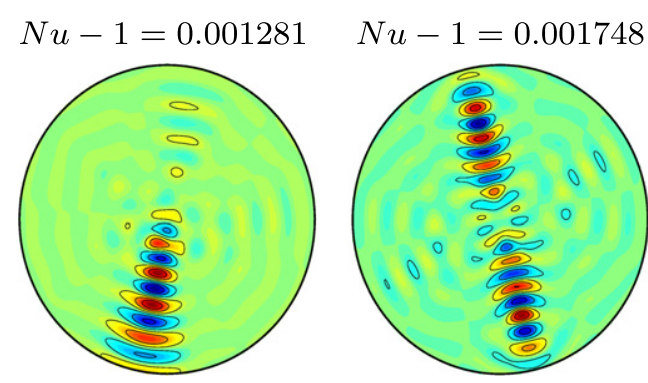

Figure 10. Contour plots of temperature at mid-height for two states in the small amplitude bursting behaviour showing the azimuthal focusing along one radius and a diameter of the cell for (left) a slightly supercritical $R(R=1919, \Gamma=10.5)$ and (right) a slightly subcritical $R(R=1914, \Gamma=11)$.

$$
R=1925, \Gamma=10.5
$$

$\Theta(z=d / 2)$
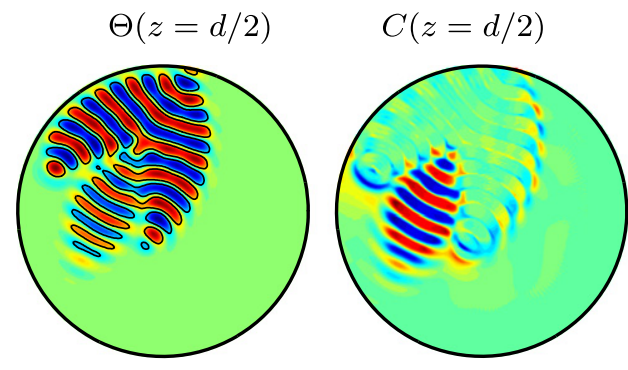

$R=1934, \Gamma=11$



Figure 11. Contour plots of temperature and concentration at mid-height for two large amplitude persistent states in the supercritical regime in the case of (left) a localized state for $R=1925, \Gamma=10.5$, and (right) a non-steady cell-filling state for $R=1934, \quad \Gamma=11$.


Figure 12. (Left) Contour plots of temperature at mid-height at several time instants during the nonlinear evolution of the solution at $R=1934, \Gamma=11$. From left to right $t=1625,1665,1705,1745$. (Right) Evolution of deviation of temperature at two points of the cell located near the lateral wall at $r=0.85 R$ (solid line) and in the bulk at $r=0.47 R$ (dashed line).

instant in figure 13, for parameters $R=1918, \Gamma=11$. In this figure we display the Nusselt number as a function of time, the contour plot of temperature at mid height, and the kinetic energy bar chart at a given time instant. In the upper part, we represent the small amplitude solution at $t=712$ obtained from the linear growth of the critical mode that exhibits the bursting behaviour. In the lower part, we show a confined state at $t=607$ obtained from the solution that almost fills the cell at $R=1934$ by decreasing the Rayleigh number. Both solutions have been computed at 

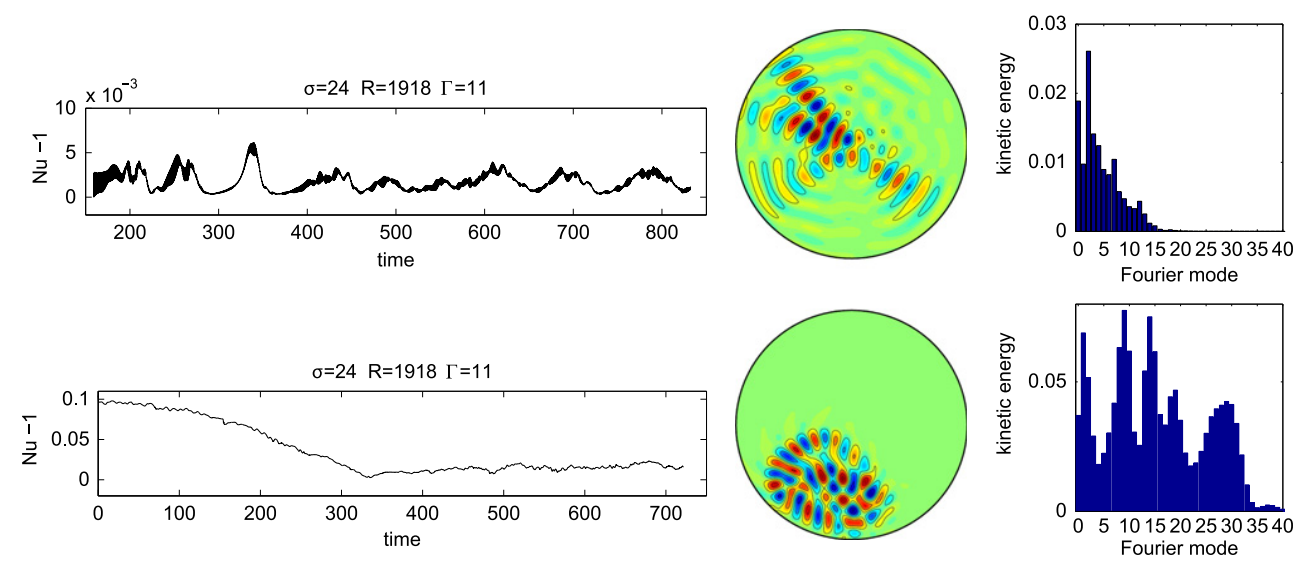

Figure 13. Nusselt number versus time, contour plot of temperature at mid-height and kinetic energy bar charts for two different solutions at $R=1918, \Gamma=11$. On the top, the solution obtained from the linear growth of the critical mode $\left(t=712, N u-1=0.138 \times 10^{-2}\right)$. On the bottom, a confined state obtained starting from a solution that initially fills the cell, and by decreasing the Rayleigh number $\left(t=607, N u-1=0.139 \times 10^{-1}\right)$.

the same Rayleigh number $R=1918$. It is important to notice the different maximum values of the deviation of temperature in this plane $(0.06,0.15)$ for the two solutions, as well as the different values of the Nusselt number $\left(\mathrm{Nu}-1=0.138 \times 10^{-2}, 0.139 \times 10^{-1}\right)$. The stronger nonlinear character of the confined solution can be appreciated by comparing the two bar charts, with a much higher amplitude and wider band of the Fourier modes.

\section{Conclusions}

In this paper we have studied pattern formation of binary mixture convection in a cylinder by means of numerically solving the three dimensional Boussinesq equations withth the Soret effect, and applying physical boundary conditions on the boundaries (no-slip perfectly conducting top and bottom lids, and perfectly insulating sidewalls). Computations are especially costly due to the narrow boundary layers in the concentration field produced by the different time scales in thermal and mass diffusion, which require large spatial resolutions to be accurately resolved and lengthy simulations. We have initiated the study with the linear stability analysis of the conduction state. As expected, the primary bifurcation for negative separation ratio mixtures is oscillatory, and we have determined, for several mixtures, the critical parameters of the onset of convection, that is, the critical values of the Rayleigh number, frequency and azimuthal wavenumber of the pattern, as a function of the aspect ratio of the cell for $0.25 \lesssim \Gamma \lesssim 3$. Our results show that the most influencing parameter in the critical Rayleigh number is the separation ratio of the mixture, and that for small aspect ratio cells, $0.25 \lesssim \Gamma \lesssim 2$, the critical azimuthal wavenumber of the pattern is essentially determined by the geometry of the cell, that is, the critical wavenumber in binary mixtures coincides with that of pure fluids provided the value of $\Gamma$ is the same. After that, we have focused on exploring the formation of the different nonlinear patterns that arise in the neighbourhood of the onset of convection for several cell sizes in the range $0.25 \lesssim \Gamma \lesssim 11$.

Different behaviour is observed depending on the value of the aspect ratio of the cell. In highly constrained geometries $\Gamma \lesssim 2$ pure thermal stationary modes are always selected despite 
the oscillatory nature of the primary bifurcation. In this case, away from the crossing points, the linear stability curves corresponding to each azimuthal mode differ substantially, and we have not observed multiplicity of stable solutions; the dominant wavenumber of the nonlinear pattern arising at the onset is that of the critical wavenumber. Also, we have not been able to obtain any stable time-dependent solution for such confined geometries near onset.

As the aspect ratio of the cell increases, the concentration field begins to play a role in the dynamics. For intermediate aspect ratio cells, in particular for a cell of aspect ratio $\Gamma=3$, the dynamics near the onset is different from the pure fluid case. Our simulations show the occurrence of at least three convective patterns partly coexisting simultaneously for equal values of the control parameter. While two of these patterns are stationary, the third one is time dependent.

In highly extended systems, $\Gamma \approx 11$, pattern formation near the onset is completely different from the pure fluid case, and a startling diversity of confined patterns is observed. The choice of parameters of our study is motivated by the configuration of the experiments of Lerman et al $(1993,1996,1999)$. With respect to the previous simulations we had done in this system (Mercader et al 2008) we have now longer time series available, obtained with larger spatial resolutions, which confirm that the fully nonlinear state that fills the cell completely is not stationary, as described in experiments, but slowly moving. Remarkably, we obtain persistent large amplitude localized states not reported previously. We have been able to describe the concentration field in these two types of large amplitude states, the localized and the extended states. Additionally, we have described an hysteretic behaviour, since we have obtained coexistence of two types of patterns, with large and small amplitude. Finally, we have not been able to obtain the localized wall-state described in Lerman et al (1993) consisting of clockwise-travelling rolls attached to the wall.

\section{Acknowledgments}

This work is funded by DGICYT under grant FIS2009-08821.

\section{References}

Alonso A, Batiste O, Meseguer A and Mercader I 2007 Complex dynamical states in binary mixture convection with weak negative soret coupling Phys. Rev. E 75026310

Barten W, Lücke M and Kamps M 1991 Localized traveling-wave convection in binary-fluid mixtures Phys. Rev. Lett. 66 2621-4

Barten W, Lücke M, Kamps M and Schmitz R 1995a Convection in binary fluid mixtures: I Phys. Rev. E $515636-61$

Barten W, Lücke M, Kamps M and Schmitz R 1995b Convection in binary fluid mixtures: II Phys. Rev. E $515662-80$

Batiste O, Mercader I, Net M and Knobloch E 1999 Onset of oscillatory binary fluid convection in finite containers Phys. Rev. E 59 6730-41

Batiste $\mathrm{O}$ and Knobloch E 2005 Simulations of localized states of stationary convection in ${ }^{3} \mathrm{He}-{ }^{4} \mathrm{He}$ mixtures Phys. Rev. Lett. 95244501

Batiste O, Knobloch E, Alonso A and Mercader I 2006 Spatially localized binary fluid convection J. Fluid Mech. 560 149-58

Boronska K and Tuckerman L 2010a Extreme multiplicity in cylindrical Rayleigh-Bénard convection. I. Time dependence and oscillations Phys. Rev. E 81036320

Boronska K and Tuckerman L 2010b Extreme multiplicity in cylindrical Rayleigh-Bénard convection. II. Bifurcation diagram and symmetry classification Phys. Rev. E 81036321

Fornberg B 1998 A Practical Guide to Pseudospectral Methods (Cambridge: Cambridge University Press) 
Hébert F, Hufschmid R, Scheel J and Ahlers G 2010 Onset of Rayleigh Bénard convection in cylindrical containers Phys. Rev. E 81046318

Hof B, Lucas G J and Mullin T 1999 Flow state multiplicity in convection Phys. Fluids 11 102815-7

Hugues S and Randriamampianina A 1998 An improved projection scheme applied to pseudospectral methods for the incompressible Navier-Stokes equations Int. J. Numer. Methods Fluids 28 501-21

Jung D and Lücke M 2002 Localized waves without the existence of extended waves: oscillatory convection of binary mixtures with strong soret effect Phys. Rev. Lett. 89054502

Kolodner P, Glazier J A and Williams H 1990 Dispersive chaos in one-dimensional traveling waveconvection Phys. Rev. Lett. 65 1579-82

Kolodner P 1992 Observations of the Eckhaus instability in one dimensional traveling wave convection Phys. Rev. A 46 1739-42

Kolodner P 1993 Coexisting traveling waves and steady rolls in binary-fluid convection Phys. Rev. E 48 R665

Kolodner P 1994 Stable, unstable and defected confined states of traveling-wave convection Phys. Rev. E 50 2731-5

Kolodner P, Slimani S, Aubry N and Lima R 1995 Characterization of dispersive chaos and related states of binary-fluid convection Physica D 85 165-224

Leong S 2002 Numerical study of Rayleigh-Bénard convection in a cylinder Numer. Heat Transfer A 41 673-831

Lerman K, Bodenschatz E, Cannell D S and Ahlers G 1993 Transient localized states in 2d binary liquid convection Phys. Rev. Lett. 703572

Lerman K, Ahlers G and Cannell D S 1996 Different convection dynamics in mixtures with the same separation ratio Phys. Rev. E 53 R2041

Lerman K, Cannell D S and Ahlers G 1999 Analysis of transients for binary mixture convection in cylindrical geometry Phys. Rev. E 59 2975-85

Lücke M, Barten W, Büchel P, Fütterer C, Hollinger St and Jung Ch 1998 Evolution of Spontaneous Structures in Dissipative Continuous Systems (Berlin: Springer) pp 127-96

Ma D J, Sun D J and Yin X Y 2006 Multiplicity of steady states in cylindrical Rayleigh-Bénard convection Phys. Rev. E 74037302

Mercader I, Net M and Falqués A 1991 Spectral methods for high order equations Comp. Meth. Appl. Mech. Eng. 91 1245-51

Mercader I, Net M and Knobloch E 1995 Binary fluid convection in a cylinder Phys. Rev. E 51 339-50

Mercader I, Alonso A and Batiste O 2008 Spatiotemporal dynamics near the onset of convection for binary mixtures in cylindrical containers Phys. Rev. E 77036313

Mercader I, Batiste O and Alonso A 2010 An efficient spectral code for incompressible flows in cylindrical geometries Comput. Fluids 39 215-24

Prat J, Massaguer J M and Mercader I 1995 Large-scale flows and resonances in 2-D thermal convection Phys. Fluids 7 121-34

Prat J, Mercader I and Knobloch E 1998 Resonant mode interactions in Rayleigh-Bénard convection Phys. Rev. E 58 3145-56

Rüdiger S and Feudel F 2000 Pattern formation in Rayleigh-Bénard convection in a cylindrical geometry Phys. Rev. E 62 4927-31

Trefethen L N 2000 Spectral Methods in Matlab (Philadelphia, PA: SIAM) 